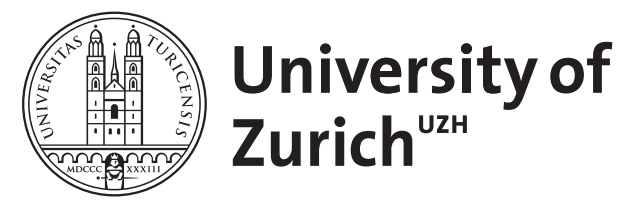

\title{
Cubature techniques for 3-D Galerkin BEM
}

\author{
Sauter, S
}

\begin{abstract}
We present cubature methods for the approximation of surface integrals arising from Galerkin discretizations of 3-D boundary integral equations. This numerical integrator is fully implicit in the sense that the form of the kernel function, the surface parametrization, the trial and test space, and the order of the singularity of the kernel functions are not used explicitly. Different kernels can be treated by just replacing the subroutine which evaluates the kernel function in certain surface points. Furthermore, the implementation of the integrator is relatively easy since it can be checked on simple test kernels as, e.g., polynomials where the exact integrals are available. We discuss the convergence of the cubature methods together with a stability and consistency analysis in order to determine the minimal cubature orders a priori.
\end{abstract}

Other titles: Proceedings of the 12th GAMM-Seminar, Kiel, Germany, January 19-21, 1996

Posted at the Zurich Open Repository and Archive, University of Zurich ZORA URL: https://doi.org/10.5167/uzh-22561

Book Section

Originally published at:

Sauter, S (1996). Cubature techniques for 3-D Galerkin BEM. In: Hackbusch, W; Wittum, G. Boundary elements: implementation and analysis of advanced algorithms (Kiel, 1996). Braunschweig: Vieweg, 29-44. 


\title{
CUBATURE TECHNIQUES FOR 3-D GALERKIN BEM
}

\author{
Stefan A. Sauter \\ Lehrstuhl für Praktische Mathematik \\ Mathematisches Seminar Bereich II \\ Christian-Albrechts-Universität zu Kiel \\ Hermann-Rodewald-Str. 3/1 \\ 24098 Kiel, Germany
}

\section{SUMMARY}

In this paper we present cubature methods for the approximation of surface integrals arising from Galerkin discretizations of 3-d boundary integral equations. This numerical integrator is fully implicit in the sense that the form of the kernel function, the surface parametrization, the trial and test space, and the order of the singularity of the kernel function is not used explicitly. Different kernels can be treated by just replacing the subroutine which evalutes the kernel function in certain surface points.

Furthermore, the implementation of the integrator is relatively easy since it can be checked on simple test kernels as, e.g., polynomials where the exact integrals are available.

We discuss the convergence of the cubature methods together with a stability and consistency analysis in order to determine the minimal cubature orders a priori.

\section{INTRODUCTION}

In this paper we will discuss Galerkin discretizations of Fredholm integral equations on two-dimensional surfaces. From the theoretical point of view as, e.g., convergence, stability, and symmetry behaviour, the Galerkin method is advantageous compared with collocation or Nyström discretizations. In practice, however, especially in the case of 2-d surfaces, the collocation or Nyström method is usually implemented. The only reason for this is that, for Galerkin discretizations, the elements of the system matrix are given by two two-dimensional integrations over pairs of surface patches which, in particular, contain a kernel function with a characteristic singular and near-singular behaviour.

In our paper we will present efficient cubature methods for the approximation of that kind of integrals. (Note that in more than one dimension the terminus quadrature is replaced by cubature). These integrators are fully automatic, which means, that they neither depend on the explicit form of the kernel function nor on the choice of the trial 
space and surface parametrization. Different kernels can be treated by just replacing the subroutine which evaluates the kernel function at certain surface points while the numerical integrator has not to be modified. In contrast to analytic and semi-analytic methods such kind of strategies have the advantage that they are relatively easy to implement and behave robustly with respect to cancellation errors. They can be tested isolated for polynomial test kernel where the exact solution is available.

The cubature strategy is based on the following idea. First all singular integrals are transformed into integrals with analytic integrands by employing regularizing coordinate transforms. These coordinate transforms were first introduced in [12] and [4] where they were combined with semi-analytic optimization techniques based on expansions of the kernel function and surface parametrization. A fully implicit treatment of such integrals for special applications as wavelets and $h p$-methods were presented in [11]. Here, we present a modified version of these coordinate transforms for the $h$-version of the Galerkin BEM together with an error analysis. Furthermore, the results of a stability and consistency analysis will be presented which allow to choose the order of integration minimally. We emphasize the importance of a proper consistency analysis for this kind of problem, since the bottleneck in any $3-\mathrm{d}$ implementation of Galerkin discretizations of boundary integrals equations is the evaluation of the four dimensional integrals defining the matrix entries. Only slight over-integration usually will lead to an increase of the CPU-time by orders of magnitude. Such a stability and consistency analysis for the regular integrals was presented in [13]. Here, we will focus on the singular and near-singular integrals which can be regarded as Part II of the mentioned paper [13].

Due the limited space in these proceedings the proofs are only sketched. We concentrate here on the practical point of view, i.e., present the cubature techniques such that they can be implemented in a straightforward manner. The full mathematical analysis and implementation details are worked out in [9].

\section{BOUNDARY INTEGRAL EQUATIONS}

We consider the variational formulation of Fredholm integral equations. Let $\Gamma$ be a piecewise analytic, orientable Lipschitz surface of a bounded domain $\Omega \subset \mathbb{R}^{3}$. Let $W^{k, p}(\Gamma)$ denote the usual Sobolev space as defined, e.g., in the book of Adams [1]. The norm of $W^{k, p}$ is denoted by $\|\cdot\|_{k, p}$ and the seminorm containing only the highest derivatives by $|\cdot|_{k, p}$. For $p=2$ we write $H^{k}$ instead of $W^{k, 2}$ and $\|\cdot\|_{k}$ instead of $\|\cdot\|_{k, 2}$.

For given right-hand side $f \in H^{s_{1}^{\prime}}$, we are seeking $u \in H^{s_{1}}(\Gamma)$ such that

$$
\left(v, \lambda_{1} u\right)_{0}+\left(v, K_{1} u\right)_{0}=\left(v, \lambda_{2} f\right)_{0}+\left(v, K_{2} f\right)_{0}, \quad \forall v \in H^{s_{1}}(\Gamma)
$$

where $(\cdot, \cdot)_{0}$ denotes the $L^{2}$-scalar product. The associated operators $\lambda_{i} I+K_{i}$ are assumed to be of order $2 s_{i}$ and explicitly given by

$$
K_{i} u:=p . f \cdot \int_{\Gamma} k_{i}(x, y) u(y) d y
$$

where the integral has to be understood in the finite part sense (see [16]) and $k_{i}$ denotes the kernel functions. The functions $\lambda_{i}: \Gamma \rightarrow \mathbb{R}$ are assumed to be piecewise constant on 
smooth patches of the surface and may jump across corners and edges. The regularity exponent $s_{1}^{\prime}$ for the right-hand side should be such that $\lambda_{2} f+K_{2} f \in H^{-s_{1}}$, i.e., $s_{1}^{\prime}=-s_{1}-$ $2 s_{2}$. The left-hand side of (1) defines the bilinear forms $a, a_{1}, a_{2}: H^{s_{1}}(\Gamma) \times H^{s_{1}}(\Gamma) \rightarrow \mathbb{C}$ as follows

$$
\begin{aligned}
a_{1}(u, v) & :=\left(v, \lambda_{1} u\right)_{0}, \\
a_{2}(u, v) & :=\left(v, K_{1} u\right)_{0}, \\
a & :=a_{1}+a_{2} .
\end{aligned}
$$

The right-hand side defines the functionals $F, F_{1}, F_{2}: H^{s_{1}} \rightarrow \mathbb{C}:$

$$
\begin{aligned}
& F_{1}(v):=\left(v, \lambda_{2} f\right)_{0}, \\
& F_{2}(v):=\left(v, K_{2} f\right)_{0}, \\
& F:=F_{1}+F_{2} .
\end{aligned}
$$

Remark 1 The order of the operator $\lambda_{i} I+K_{i}$ can be negative which implies in particular $\lambda_{i} \equiv 0$.

Throughout this paper we assume that the bilinear form $a$ is continuous

$$
|a(u, v)| \leq C_{a}\|u\|_{s_{1}}\|v\|_{s_{1}}, \quad \forall u, v \in H^{s_{1}}(\Gamma)
$$

and elliptic

$$
\|a(u, u)\| \geq C_{e}\|u\|_{s_{1}}^{2}-C_{c}\|u\|_{t}^{2} \quad \forall u \in H^{s_{1}}(\Gamma)
$$

with suitable $t<s_{1}$. For simplicity, we assume that (1) has a unique solution for every $f \in$ $H^{s_{1}^{\prime}}(\Gamma)$. Otherwise, one has to introduce suitable normalizations or to restrict $H^{s_{i}}(\Gamma)$ to some quotient space. Then, the theory presented below directly applies to these situations.

The following model problem illustrates the abstract setting.

Example 2 Consider the Laplace equation in the exterior of the bounded domain $\Omega \subset \mathbb{R}^{3}$.

$$
\begin{array}{ll}
\Delta u=0, & \text { in } \mathbb{R}^{3} \backslash \bar{\Omega}, \\
\frac{\partial u}{\partial n}=f, & \text { on } \Gamma:=\partial \Omega, \\
u(x) \rightarrow 0, & \|x\| \rightarrow \infty,
\end{array}
$$

where $n(x)$ denotes the normal in $x \in \Gamma$ pointing into $\Omega$ and $\partial / \partial n:=\langle n, \nabla \cdot\rangle$. Let the bilinear form $a: H^{1 / 2} \times H^{1 / 2} \rightarrow \mathbb{R}$ be defined by

$$
a(u, v):=\int_{\Gamma} v(x) p \cdot f \cdot \int_{\Gamma} u(y) \frac{\partial}{\partial n_{x}} \frac{\partial}{\partial n_{y}} \frac{1}{\|x-y\|} d y d x
$$

and the functional of the right-hand side by

$$
F(v):=\frac{1}{2}(v, f)_{0}-\int_{\Gamma} v(x) p \cdot f \cdot \int_{\Gamma} f(y) \frac{\partial}{\partial n_{x}} \frac{1}{\|x-y\|} d y d x
$$

Let $f$ be such that $F \in\left(H^{1 / 2} / \mathbb{R}\right)^{\prime}$. Then the problem of finding $u \in H^{1 / 2} / \mathbb{R}$ such that

$$
a(u, v)=F(v), \quad \forall v \in\left(H^{1 / 2} / \mathbb{R}\right)^{\prime}
$$

has a unique solution. This function $u$ is the trace of the solution of (5). 


\section{GALERKIN BOUNDARY ELEMENT METHODS}

Let $\Omega$ be a three-dimensional domain with piecewise analytic, orientable Lipschitz surface $\Gamma$. We assume that $\Gamma$ is a smooth image of an interpolating polyhedron. To be more concrete, let $\tilde{\Gamma}$ be a surface of a polyhedron consisting of plane faces $\tilde{\Gamma}_{j}$ such that there exists a bijective mapping $\eta: \tilde{\Gamma} \rightarrow \Gamma$ with the following properties.

1. $\eta$ is bi-Lipschitz continuous, i.e.,

$$
C_{1}\|\tilde{x}-\tilde{y}\| \leq\|\eta(\tilde{x})-\eta(\tilde{y})\| \leq C_{2}\|\tilde{x}-\tilde{y}\|, \quad \forall \tilde{x}, \tilde{y} \in \tilde{\Gamma},
$$

2. there exists plane extensions $\tilde{\Gamma}_{j}^{\star}$ of $\tilde{\Gamma}_{j}$, i.e., $\tilde{\Gamma}_{j} \subset \subset \tilde{\Gamma}_{j}^{\star}$ where $\left.\eta\right|_{\tilde{\Gamma}_{j}}$ can be extended analytically.

In this paper we restrict the presentation to triangular elements. Generalizations to the quadrilateral case are straightforward by using the techniques presented in [15] and [14]. Let $\tilde{\tau}:=\left\{\tilde{K}_{1}, \tilde{K}_{2}, \ldots, \tilde{K}_{N}\right\}$ denote a grid of the surface $\tilde{\Gamma}$ consisting of plane triangles having the property that

$$
\begin{aligned}
& \tilde{\Gamma}:=\varlimsup_{\tilde{K} \in \tilde{\tau}} \tilde{K}, \\
& \tilde{K} \cap \tilde{K}^{\prime}=\emptyset, \quad \forall \tilde{K}, \tilde{K}^{\prime} \in \tau \text { with } \tilde{K} \neq \tilde{K}^{\prime} .
\end{aligned}
$$

This grid induces a grid on the true surface $\Gamma$ by $\tau:=\{\eta(\tilde{K})\}_{\tilde{K} \in \tilde{\tau}}$. Note that the transformation $\eta$ is independent of the grid $\tilde{\tau}$.

In order to define finite element spaces on $\Gamma$, we lift polynomial spaces on a reference element onto the surface elements. In this light, we define, for a domain $D \subset \mathbb{R}^{d}$, the space of polynomials of total degree $p$ by

$$
\Pi_{p}(D):=\left\{v(x)=\sum_{|\alpha| \leq p} c_{\alpha} x^{\alpha} \text { for some } c_{\alpha} \in \mathbb{C}\right\},
$$

where $\alpha \in \mathbb{N}_{0}^{d}$ is a multi-index and $|\alpha|$ denotes the $l^{1}$-norm.

For triangular elements we employ the master element

$$
K^{0}:=\left\{\hat{x} \in \mathbb{R}_{+}^{2} \mid 0 \leq \hat{x}_{1} \leq 1,0 \leq \hat{x}_{2} \leq \hat{x}_{1}\right\}
$$

and the polynomials of degree $p$ are given by $\Pi_{p}\left(K^{0}\right)$. The transformation of $K^{0}$ onto $\tilde{K}$ is affine linear and denoted by $\kappa_{\tilde{K}}$ while the mapping on the true surface element is given by $\kappa_{K}:=\eta \circ \kappa_{\tilde{K}}$. The space $\Pi_{p}\left(K^{0}\right)$ is lifted onto the surface patch $K$ by $\kappa_{K}$ :

$$
\Pi_{p}(K):=\left\{u: K \rightarrow \mathbb{C} \mid u \circ \kappa_{K} \in \Pi_{p}\left(K^{0}\right)\right\} .
$$

The global finite element space is given by

$$
V_{k, p}:=\left\{u \in \mathcal{C}^{k}(\Gamma)|\forall K \in \tau: u|_{K} \in \Pi_{p}(K)\right\} .
$$


The Galerkin boundary element discretization to (1) is characterized by replacing the infinite dimensional Sobolev spaces $H^{s_{1}}$ by the finite dimensional subspace $V_{k, p}$ : for given $f \in H^{s_{1}^{\prime}}$, we are seeking $u_{G} \in V_{k, p}$ such that

$$
a\left(u_{G}, v\right)=F(v), \quad \forall v \in V_{k, p} .
$$

In this paper we are only considering conforming methods, i.e., the exponent $k$ in (8) has to be chosen such that $V_{k, p} \subset H^{s_{1}}$.

In order to compute the solution to problem (9), we introduce a basis $\left\{\varphi_{i}\right\}_{1<i \leq N}$ of $V_{k, p}$. Any function $u \in V_{k, p}$ has a unique basis representation

$$
u(x)=\sum_{i=1}^{n} \mathbf{u}_{i} \varphi_{i}(x)
$$

For $1 \leq i, j \leq N$, let the system matrix $\mathbf{K}$ and the vector of the right-hand side be defined by

$$
\begin{aligned}
\mathbf{K}_{i, j}: & =a\left(\varphi_{i}, \varphi_{j}\right), \\
\mathbf{F}_{i} & :=F\left(\varphi_{i}\right) .
\end{aligned}
$$

Then the solution of

$$
\mathbf{K} \mathbf{u}_{G}=\mathbf{F}
$$

is linked to the solution of (9) by

$$
u_{G}(x)=\sum_{i=1}^{N} \mathbf{u}_{G, i} \varphi_{i}(x) .
$$

For the computation of the matrix $\mathbf{K}$ and right-hand side $\mathbf{F}$, integrals over (pairs of) surface patches have to be evaluated. This can be done exactly only in very special situations. In general, one has to use numerical cubature techniques to approximates the coefficients. The effect of numerical integration is considered as an approximation of the continuous (bi-)linear forms. The bilinear forms $\tilde{a}, \tilde{a}_{1}$, and $\tilde{a}_{2}$ are defined by replacing the integrals by numerical cubature, while the definition of $\tilde{F}, \tilde{F}_{1}$ and $\tilde{F}_{2}$ is analogously. Note that these approximate (bi-)linear forms have to be defined only on the discrete level, i.e., for functions in $V_{k, p}$ and not necessarily for functions in $H^{s_{1}}(\Gamma)$.

The Galerkin discretization including numerical cubature is given by seeking $\tilde{u}_{G} \in V_{k, p}$ such that

$$
\tilde{a}\left(\tilde{u}_{G}, v\right)=\tilde{F}(v), \quad \forall v \in V_{k, p} .
$$

In the next section we will present families of cubature methods which approximates the continuous (bi-)linear forms to any desired accuracy. Then, we will estimate the cubature error which, in combination with a stability and consistency analysis, enables us to determine the minimal cubature orders a priori. 


\section{KERNEL PROPERTIES OF BOUNDARY INTEGRAL EQUATIONS}

Before presenting the cubature methods we have to collect the characteristic properties of the kernel functions. The kernel functions are suitable Gâteau derivatives of the fundamental solution of the underlying boundary value problem. The following properties are satisfied for all common kernels which arise by transforming elliptic boundary value problems in $\mathbb{R}^{3}$ into integral equations (see [16]). We formulate these conditions in a way which will turn out to be convenient for the cubature analysis. We assume that $k(x, y)$ can be written in the form

$$
k(x, y)=\sum_{|\alpha| \geq t} s_{\alpha}(x, y) \frac{(y-x)^{\alpha}}{\|y-x\|^{\sigma+t}}
$$

with functions $s_{\alpha}: \Gamma \times \Gamma \rightarrow \mathbb{C}$ being analytic on pairs of smooth surface patches of $\Gamma$. The integer $\sigma$ denotes the order of the singularity and we assume that $\sigma \leq 3$ holds. For $\sigma \leq 2$, we assume that the kernel is anti-symmetric, i.e.,

$$
k(x, y)+k(y, x)=\sum_{|\alpha| \geq t+1} \tilde{s}_{\alpha}(x, y) \frac{(y-x)^{\alpha}}{\|y-x\|^{\sigma+t}} .
$$

This condition is stronger than the common Tricomi-Giraud-Mikhlin condition and implies that the kernel is Cauchy-singular, i.e.,

$$
\lim _{\varepsilon \rightarrow 0} \int_{K \backslash B_{\varepsilon}(x)} k(x, y) f(y) d y
$$

exists for all elements $K \in \tau$, Lipschitz-continuous functions $f \in C^{0,1}(K)$, and $x \in \Gamma \backslash \partial K$.

For $\sigma=3$, the kernel function is called hyper-singular and an appropriate regularization has to be performed on the continuous level. We assume that the bilinear form $a_{2}$ of (2) can be rewritten as follows

$$
a_{2}(u, v)=\sum_{i=0}^{m_{1}} \sum_{j=0}^{m_{2}} \int_{\Gamma} \int_{\Gamma} D_{i}[v](x, y) k_{i, j}^{r e g}(x, y) D_{j}[u](x, y) d y d x
$$

with $m_{1,2} \in\{0,1\}$. The operators $D_{i}$ are of order $i$ satisfying

$$
\left\|D_{i}[w](x, y)\right\|_{0} \leq C\|w\|_{i}
$$

while the kernel functions $k_{i, j}^{r e g}$ satisfy (11) and (12) with $\sigma_{i, j}^{r e g} \leq 2$. Furthermore, throughout the paper, we assume the relations $\sigma_{i, j}^{r e g}+i+j \leq 3$ and $s_{1} \leq m_{1} \leq p$. An analogous representation has to be employed if the kernel function $k_{2}$ is hyper-singular. For weakly or Cauchy singular kernels we put $k_{0,0}^{r e g}=k$ and $m_{1}=m_{2}=0$. 
Example 3 The bilinear form a $(u, v)$ of example (D) can be rewritten as follows

$$
a(u, v)=\int_{\Gamma} \int_{\Gamma} D_{1}[v](x, y) D_{1}[u](x, y) k^{r e g}(x, y) d y d x
$$

with $D_{1}[w](x, y):=(w(y)-w(x)) /\|y-x\|$ and

$$
k^{r e g}(x, y):=\|y-x\|^{2} \frac{\partial^{2}}{\partial n_{x} \partial n_{y}} \frac{1}{\|x-y\|} .
$$

The following representation is proved in [10])

$$
a(u, v):=-\int_{\Gamma}\left(-n(x) \times \nabla_{x}\right) u(x) \int_{\Gamma} \frac{1}{\|x-y\|}\left(n(y) \times \nabla_{y}\right) v(y) d y d x .
$$

Using this kind of regularization it is natural to assume in the following that the bilinear form has a representation with a Cauchy-singular kernel function $(\sigma \leq 2)$ while, possibly, the functions $u$ and $v$ have to replaced by $D_{j}[\cdot]$. We state that for much more general kernel functions such representation are available, too (see [10], [6], [7], [5], [3]). Another approach is presented in [8] which can be treated by a slight modification of the cubature techniques below.

We are now ready for the presentation of the cubature techniques.

\section{CUBATURE TECHNIQUES FOR GALERKIN DISCRETIZATIONS}

In view of the previous section, the (bi-) linear forms can be decomposed as follows

$$
\begin{array}{ll}
a_{1}(u, v)=\sum_{K \in \tau} a_{K}(u, v) & a_{2}(u, v)=\sum_{K_{x} \times K_{y} \in \tau \times \tau} a_{K_{x} \times K_{y}}(u, v) \\
F_{1}(v)=\sum_{K \in \tau} F_{K}(v) & F_{2}(v)=\sum_{K_{x} \times K_{y} \in \tau \times \tau} F_{K_{x} \times K_{y}}(v)
\end{array}
$$

with

$$
\begin{array}{ll}
a_{K}(u, v):=\int_{K} \lambda_{1} u v d x, & a_{K_{x} \times K_{y}}(u, v):=\lim _{\varepsilon \rightarrow 0} \int_{K_{x} \times K_{y}} A(x, y) k_{1}(x, y) d y d x, \\
F_{K}(v):=\int_{K} \lambda_{2} f v d x, & F_{2}(v):=\lim _{\varepsilon \rightarrow 0} \int_{K_{x} \times K_{y}} B(x, y) k_{2}(x, y) d y d x . \\
\|x-y\| \geq \varepsilon
\end{array}
$$

The functions $A$ and $B$ are given by $A(x, y)=D_{1}[u](x, y) D_{2}[v](x, y)$ and $B(x, y):=$ $D_{3}[f](x, y) D_{4}[v](x, y)$ with the operators $D_{j}$ of order $m_{j}$ defined in the previous section. The kernel functions $k_{i}$ are assumed to satisfy the assumptions of the previous section with $\sigma \leq 2$.

In the first part we will present coordinate transforms which render the integrands above analytic such that they can be treated by standard cubature methods. We emphasize that these transformations does not contain any approximation process.

The integrands of the integrals corresponding to $a_{K}$ and $F_{K}$ are analytic and can be approximated by standard techniques. In the following we will concentrate on the integrals $a_{2}$ and $F_{2}$. Here, the integrands have a singular, near-singular, or regular behaviour which requires different transformations. The following four basis cases have to be distinguished. 
1. "Identical Panels": $K_{x}=K_{y}$.

2. "Common Edge": $K_{x}$ and $K_{y}$ share exactly an edge.

3. "Common Vertex": $K_{x}$ and $K_{y}$ share exactly a vertex.

4. "Regular Case": $\bar{K}_{x}$ and $\bar{K}_{y}$ are disjoint.

Condition (6) does not guarantee that every pair $K_{x}, K_{y} \in \tau$ satisfies one of the four cases listed above. However, it is clear that one of the panels $K_{x}, K_{y}$ can be subdivided such that the arising sub-panels satisfy one of the conditions above. The methods presented below then have to be applied to the sub-panels. Hence, we assume for the following that any pair of panels $K_{x} \times K_{y}$ satisfies one and only one of the conditions above.

In the following we will discuss these four cases in detail.

\section{THE CASE OF IDENTICAL PANELS}

Here, we consider integrals of the type

$$
I_{i}:=\lim _{\varepsilon \rightarrow 0} \int_{\substack{K \times K \\\|x-y\| \geq \varepsilon}} H(x, y) k(x, y) d y d x
$$

with $H$ is either the function $A$ or $B$ defined above. The kernel function $k$ is assumed to satisfy the assumptions on the kernel functions with $\sigma \leq 2$. We transform this integral onto the scaled master element $\Delta^{h}:=h \Delta^{0}$ by the transformation $\kappa_{K}^{h}(\hat{x}):=\kappa_{K}(\hat{x} / h)$ with $\kappa_{K}$ defined by $(7)$ and $h:=\operatorname{diam} K$. In local coordinates, the integrand is given by

$$
\begin{aligned}
k_{l o c}(\hat{x}, \hat{y}) & :=k\left(\kappa_{K}^{h}(\hat{x}), \kappa_{K}^{h}(\hat{y})\right) \\
H_{l o c}(\hat{x}, \hat{y}): & =g_{K}(\hat{x}) g_{K}(\hat{y}) H\left(\kappa_{K}^{h}(\hat{x}), \kappa_{K}^{h}(\hat{y})\right)
\end{aligned}
$$

with

$$
g_{K}(\hat{x}):=\left|\left\{\left\langle\frac{\partial \kappa_{K}^{h}(\hat{x})}{\partial \hat{x}_{i}}, \frac{\partial \kappa_{K}^{h}(\hat{x})}{\partial \hat{x}_{j}}\right\rangle\right\}_{1 \leq i, j \leq 2}\right|^{1 / 2} .
$$

In local coordinates the integral $I_{i}$ takes the form

$$
I_{i}:=\lim _{\varepsilon \rightarrow 0} \int_{0}^{h} \int_{0}^{\hat{x}_{1}} \int_{0}^{h} \int_{0}^{h} \hat{y}_{1}^{\hat{y}_{1}} H_{l o c}(\hat{x}, \hat{y}) k_{l o c}(\hat{x}, \hat{y}) d \hat{y} d \hat{x} .
$$

The problem with this integral is that the location of the singularity is not fixed and cannot be cancelled by degenerate coordinate transforms as, e.g., polar coordinates. The purpose of the following coordinate transforms is to fix the singularity by introducing relative coordinates as proposed in [12] and [4]. The variable $\hat{y}$ is replaced by $\hat{y}=z+\hat{x}$ and hence, the singularity of the kernel function is fixed at the origin $z=\hat{y}-\hat{x}=0$. 
The domain of the $(\hat{x}, z)$-integration can be described by the union of the following six domains

$$
\begin{array}{cl}
D_{1}=\left\{\begin{array}{c}
-h \leq z_{1} \leq 0, \\
-h \leq z_{2} \leq z_{1}, \\
-z_{2} \leq \hat{x}_{1} \leq h, \\
-z_{2} \leq \hat{x}_{2} \leq \hat{x}_{1} .
\end{array}\right\} & D_{2}=\left\{\begin{array}{c}
0 \leq z_{1} \leq h, \\
z_{1}-h \leq z_{2} \leq 0, \\
-z_{2} \leq \hat{x}_{1} \leq h-z_{1}, \\
-z_{2} \leq \hat{x}_{2} \leq \hat{x}_{1} .
\end{array}\right\} \\
D_{3}=\left\{\begin{array}{c}
0 \leq z_{1} \leq 0, \\
z_{1} \leq z_{2} \leq 0, \\
-z_{1} \leq \hat{x}_{1} \leq h, \\
-z_{2} \leq \hat{x}_{2} \leq z_{1}+\hat{x}_{1}-z_{2} .
\end{array}\right\} & D_{4}=\left\{\begin{array}{c}
\leq z_{2} \leq h, \\
-h \leq z_{1} \leq 0, \\
z_{2}-z_{1} \leq \hat{x}_{1} \leq h-z_{1}, \\
0 \leq \hat{x}_{2} \leq z_{1}+\hat{x}_{1}-z_{2} .
\end{array}\right\} \\
D_{5}=\left\{\begin{array}{c}
0 \leq z_{1} \leq h, \\
0 \leq z_{2} \leq z_{1}+h, \\
z_{2}-z_{1} \leq \hat{x}_{1} \leq h, \\
0 \leq \hat{x}_{2} \leq z_{1}+\hat{x}_{1}-z_{2} .
\end{array}\right\} & D_{6}=\left\{\begin{array}{c}
0 \leq z_{2} \leq z_{1}, \\
0 \leq \hat{x}_{1} \leq h-z_{1}, \\
0 \leq \hat{x}_{2} \leq \hat{x}_{1} .
\end{array}\right\}
\end{array}
$$

Hence, the integral $I_{i}$ takes the form

$$
I_{i}=\lim _{\varepsilon \rightarrow 0} \sum_{j=1}^{6} \int_{\substack{D_{j} \\\|z\| \geq \varepsilon}} H_{l o c}(\hat{x}, z+\hat{x}) k_{l o c}(\hat{x}, z+\hat{x}) d \hat{x} d z .
$$

Let $T_{d}^{h}$ denote the scaled $d$-dimensional unit simplex

$$
T_{d}^{h}:=\left\{\left.\hat{x} \in \mathbb{R}_{+}^{d}|| \hat{x}\right|_{l^{1}}<h\right\}
$$

and $R_{d}:=(0,1)^{d}$. The following coordinate transforms map $\left(\eta, w_{1}, w_{2}, w_{3}\right) \in R_{1} \times T_{3}^{h}$ onto the domain $D_{j}$.

$$
\begin{aligned}
& \left(\begin{array}{c}
z_{1}^{(1)} \\
z_{2}^{(1)} \\
\hat{x}_{1}^{(1)} \\
\hat{x}_{2}^{(1)}
\end{array}\right)=\left(\begin{array}{c}
-w_{1} \eta \\
-w_{1} \\
w_{1}+w_{2}+w_{3} \\
w_{1}+w_{2}
\end{array}\right), \quad\left(\begin{array}{c}
z_{1}^{(2)} \\
z_{2}^{(2)} \\
\hat{x}_{1}^{(2)} \\
\hat{x}_{2}^{(2)}
\end{array}\right)=\left(\begin{array}{c}
w_{1} \eta \\
w_{1}(\eta-1) \\
w_{1}(1-\eta)+w_{2}+w_{3} \\
w_{1}(1-\eta)+w_{2}
\end{array}\right), \\
& \left(\begin{array}{c}
z_{1}^{(3)} \\
z_{2}^{(3)} \\
\hat{x}_{1}^{(3)} \\
\hat{x}_{2}^{(3)}
\end{array}\right)=\left(\begin{array}{c}
-w_{1} \\
-w_{1} \eta \\
w_{1}+w_{2}+w_{3} \\
w_{1} \eta+w_{2}
\end{array}\right), \quad\left(\begin{array}{c}
z_{1}^{(4)} \\
z_{2}^{(4)} \\
\hat{x}_{1}^{(4)} \\
\hat{x}_{2}^{(4)}
\end{array}\right)=\left(\begin{array}{c}
w_{1} \eta \\
w_{1} \\
w_{1}(1-\eta)+w_{2}+w_{3} \\
w_{2}
\end{array}\right), \\
& \left(\begin{array}{c}
z_{1}^{(5)} \\
z_{2}^{(5)} \\
\hat{x}_{1}^{(5)} \\
\hat{x}_{2}^{(5)}
\end{array}\right)=\left(\begin{array}{c}
-w_{1} \eta \\
w_{1}(1-\eta) \\
w_{1}+w_{2}+w_{3} \\
w_{2}
\end{array}\right), \quad\left(\begin{array}{c}
z_{1}^{(6)} \\
z_{2}^{(6)} \\
\hat{x}_{1}^{(6)} \\
\hat{x}_{2}^{(6)}
\end{array}\right)=\left(\begin{array}{c}
w_{1} \\
w_{1} \eta \\
w_{2}+w_{3} \\
w_{2}
\end{array}\right) \text {. }
\end{aligned}
$$

The determinant of the Jacobi matrix equals $w_{1}$ and thus, the integral $I_{i}$ can be written in the form

$$
I_{i}=\int_{R_{1}} \int_{T_{3}^{h}} w_{1} \sum_{j=1}^{6} H_{l o c}\left(\hat{x}^{(j)}, z^{(j)}+\hat{x}^{(j)}\right) k_{l o c}\left(\hat{x}^{(j)}, z^{(j)}+\hat{x}^{(j)}\right) d w d \eta .
$$


It was shown in [12], [11], [15] that the integrand above is analytic. This implies that standard cubature techniques with properly chosen orders approximates (13) to any required accuracy.

\section{THE CASE OF A COMMON EDGE}

In this section we consider the computation of

$$
I_{e}:=\int_{K_{1} \times K_{2}} H(x, y) k(x, y) d y d x
$$

The transformations in the case of a common edge are similar as for identical panels. However, the character of the singularity slightly changes. Let $K_{1}, K_{2}$ be two surface triangles which share exactly one edge. Let the parameter domains are given by $\Delta_{1}^{h}:=\Delta^{h}$ and $\Delta_{2}^{h}:=\left\{\hat{y} \in \mathbb{R}^{2} \mid\left(\hat{y}_{1},-\hat{y}_{2}\right)^{T} \in \Delta^{h}\right\}$. Let the mapping $\kappa: \overline{\Delta_{1}^{h} \cup \Delta_{2}^{h}} \rightarrow \overline{K_{1} \cup K_{2}}$ be biLipschitz continuous. For $\hat{x} \in \Delta_{1}^{h}$ and $\hat{y} \in \Delta_{2}^{h}$, the integrand in local coordinates is given by

$$
\begin{aligned}
k_{l o c}(\hat{x}, \hat{y}): & =k(\kappa(\hat{x}), \kappa(\hat{y})) \\
H_{l o c}(\hat{x}, \hat{y}): & =H(\kappa(\hat{x}), \kappa(\hat{y})) g_{K_{1}}(\hat{x}) g_{K_{2}}(\hat{y})
\end{aligned}
$$

and $I_{e}$ takes the form

$$
I_{e}:=\int_{0}^{h} \int_{0}^{\hat{x}_{1}} \int_{0}^{h} \int_{-\hat{y}_{1}}^{0} H_{l o c}(\hat{x}, \hat{y}) k_{l o c}(\hat{x}, \hat{y}) d \hat{y} d \hat{x} .
$$

Due to the fact that $\kappa$ is bi-Lipschitz continuous, the kernel function behaves singular if and only if $\hat{x}=\hat{y}$, i.e., $\hat{x}_{1}-\hat{y}_{1}=\hat{x}_{2}=\hat{y}_{2}=0$. The location of the singularity is fixed by introducing one-dimensional relative coordinates $\hat{y}_{1}=z_{1}+\hat{x}_{1}, \hat{y}_{2}=z_{2}, \hat{x}_{2}=z_{3}$. The integration domain can be described as the union of the following six four-dimensional domains

$$
\begin{aligned}
& D_{1}=\left\{\begin{array}{c}
0 \leq z_{3} \leq h \\
-h \leq z_{1} \leq-z_{3} \\
-h-z_{1} \leq z_{2} \leq 0 \\
-z_{1}-z_{2} \leq \hat{x}_{1} \leq h
\end{array}\right\}, \quad D_{2}=\left\{\begin{array}{c}
0 \leq z_{3} \leq h \\
-z_{3} \leq z_{1} \leq 0 \\
-h-z_{1} \leq z_{2} \leq-z_{3}-z_{1} \\
-z_{1}-z_{2} \leq \hat{x}_{1} \leq h
\end{array}\right\}, \\
& D_{3}=\left\{\begin{array}{c}
0 \leq z_{3} \leq h \\
0 \leq z_{3} \leq h \\
-z_{3} \leq z_{1} \leq 0 \\
-z_{3}-z_{1} \leq z_{2} \leq 0 \\
z_{3} \leq \hat{x}_{1} \leq h \\
0 \leq z_{3} \leq h
\end{array}\right\}, \quad D_{4}=\left\{\begin{array}{c}
0 \leq z_{1} \leq h-z_{3} \\
-h \leq z_{2} \leq-z_{3}-z_{1} \\
-z_{2}-z_{1} \leq \hat{x}_{1} \leq h-z_{1}
\end{array}\right\}, \\
& D_{5}=\left\{\begin{array}{c}
0 \leq z_{3} \leq h \\
0 \leq z_{1} \leq h-z_{3} \\
0 \leq z_{1} \leq h-z_{3} \\
-z_{3} \leq z_{2} \leq 0 \\
z_{3} \leq z_{1} \leq z_{2} \leq-z_{3}-z_{1}
\end{array}\right\},
\end{aligned}
$$

The integral in these coordinates has the representation

$$
I_{e}:=\sum_{j=1}^{6} \int_{D_{j}} H_{l o c}\left(\left(\begin{array}{c}
\hat{x}_{1} \\
z_{3}
\end{array}\right),\left(\begin{array}{c}
z_{1}+\hat{x}_{1} \\
z_{2}
\end{array}\right)\right) k_{l o c}\left(\left(\begin{array}{c}
\hat{x}_{1} \\
z_{3}
\end{array}\right),\left(\begin{array}{c}
z_{1}+\hat{x}_{1} \\
z_{2}
\end{array}\right)\right) d \hat{x}_{1} d z
$$


The following coordinate transforms map the coordinates $(\eta, w) \in R_{2} \times T_{2}^{h}$ onto the domain of integration.

$$
\begin{aligned}
& \left(\begin{array}{c}
\hat{z}_{1}^{(1)} \\
z_{2}^{(1)} \\
z_{3}^{(1)} \\
\hat{x}_{1}^{(1)}
\end{array}\right)=\left(\begin{array}{c}
-w_{1} \eta_{1} \\
w_{1}\left(\eta_{1}-1\right) \\
w_{1} \eta_{2} \eta_{1} \\
w_{1}+w_{2}
\end{array}\right), \quad\left(\begin{array}{c}
z_{1}^{(2)} \\
z_{2}^{(2)} \\
z_{3}^{(2)} \\
\hat{x}_{1}^{(2)}
\end{array}\right)=\left(\begin{array}{c}
-w_{1} \eta_{2} \eta_{1} \\
w_{1}\left(\eta_{2} \eta_{1}-1\right) \\
w_{1} \eta_{1} \\
w_{1}+w_{2}
\end{array}\right), \\
& \left(\begin{array}{c}
\hat{z}_{1}^{(3)} \\
z_{2}^{(3)} \\
z_{3}^{(3)} \\
\hat{x}_{1}^{(3)}
\end{array}\right)=\left(\begin{array}{c}
w_{1}\left(\eta_{1}-1\right) \\
-w_{1} \eta_{2} \eta_{1} \\
w_{1} \\
w_{1}+w_{2}
\end{array}\right), \quad\left(\begin{array}{c}
\hat{z}_{1}^{(4)} \\
z_{2}^{(4)} \\
z_{3}^{(4)} \\
\hat{x}_{1}^{(4)}
\end{array}\right)=\left(\begin{array}{c}
w_{1}\left(\eta_{1}-\eta_{2} \eta_{1}\right) \\
-w_{1} \\
w_{1} \eta_{2} \eta_{1} \\
w_{1}\left(1-\eta_{1}+\eta_{2} \eta_{1}\right)+w_{2}
\end{array}\right), \\
& \left(\begin{array}{c}
\hat{z}_{1}^{(5)} \\
z_{2}^{(5)} \\
z_{3}^{(5)} \\
\hat{x}_{1}^{(5)}
\end{array}\right)=\left(\begin{array}{c}
w_{1}\left(1-\eta_{2} \eta_{1}\right) \\
-w_{1} \eta_{1} \\
w_{1} \eta_{2} \eta_{1} \\
w_{1} \eta_{2} \eta_{1}+w_{2}
\end{array}\right), \quad\left(\begin{array}{c}
\hat{z}_{1}^{(6)} \\
z_{2}^{(6)} \\
z_{3}^{(6)} \\
\hat{x}_{1}^{(6)}
\end{array}\right)=\left(\begin{array}{c}
w_{1}\left(1-\eta_{1}\right) \\
-w_{1} \eta_{2} \eta_{1} \\
w_{1} \eta_{1} \\
w_{1} \eta_{1}+w_{2}
\end{array}\right) .
\end{aligned}
$$

The determinant of the Jacobi matrix is given for any of the transformations above by $w_{1}^{2}$. Analogously as in [12], [11], [15] one shows that the integrand of the following representation of $I_{e}$ is analytic.

$$
I_{e}:=\int_{R_{2}} \int_{T_{2}^{h}} \eta_{1} w_{1}^{2} \sum_{j=1}^{6} H_{l o c}\left(\left(\begin{array}{c}
\hat{x}_{1}^{(j)} \\
z_{3}^{(j)}
\end{array}\right)\right)\left(\begin{array}{c}
z_{1}^{(j)}+\hat{x}_{1}^{(j)} \\
z_{2}^{(j)}
\end{array}\right) k_{l o c}\left(\left(\begin{array}{c}
\hat{x}_{1}^{(j)} \\
z_{3}^{(j)}
\end{array}\right)\right)\left(\begin{array}{c}
z_{1}^{(j)}+\hat{x}_{1}^{(j)} \\
z_{2}^{(j)}
\end{array}\right) d w d \eta
$$

Again, this integral can be approximated to any desired accuracy by standard cubature methods with properly chosen order.

Finally, we have to discuss the case that the panels have a common vertex. This is done in the following section.

\section{THE CASE OF A COMMON VERTEX}

Let $K_{1}, K_{2}$ be two surface triangles which have exactly one common point. The reference domains are given by $\Delta_{1}^{h}:=\Delta^{h}$ and $\Delta_{2}^{h}:=\left\{\hat{y} \in \mathbb{R}^{2} \mid-\hat{y} \in \Delta^{h}\right\}$. By our assumptions on the surface and the triangulation we may assume that there is a bi-Lipschitz-continuous mapping $\kappa: \overline{\Delta_{1}^{h} \cup \Delta_{2}^{h}} \rightarrow \overline{K_{1} \cup K_{2}}$ which is analytic on each reference element. The integral in local coordinates is given by

$$
I_{p}=\int_{0}^{h} \int_{0}^{\hat{x}_{1}} \int_{-h}^{0} \int_{\hat{y}_{1}}^{0} H_{l o c}(\hat{x}, \hat{y}) k_{l o c}(\hat{x}, \hat{y}) d \hat{y} d \hat{x}
$$

where we adopt the notations of the previous section. We split the domain of integration according to

$I_{p}=\int_{0}^{h} \int_{0}^{\hat{x}_{1}} \int_{-\hat{x}_{1}}^{0} \int_{\hat{y}_{1}}^{0} H_{l o c}(\hat{x}, \hat{y}) k_{l o c}(\hat{x}, \hat{y}) d \hat{y} d \hat{x}+\int_{-h}^{0} \int_{\hat{y}_{1}}^{0} \int_{0}^{-\hat{y}_{1}} \int_{0}^{\hat{x}_{1}} H_{l o c}(\hat{x}, \hat{y}) k_{l o c}(\hat{x}, \hat{y}) d \hat{x} d \hat{y}$. 
The following transformations maps $\left(\eta_{1}, \eta_{2}, \eta_{3}, w\right) \in R_{3} \times T_{1}^{h}$ onto the domains of integration.

$$
\left(\begin{array}{c}
\hat{x}_{1}^{(1)} \\
\hat{x}_{2}^{(1)} \\
\hat{y}_{1}^{(1)} \\
\hat{y}_{2}^{(1)}
\end{array}\right)=\left(\begin{array}{c}
w \\
\eta_{2} w \\
-\eta_{1} w \\
-\eta_{3} \eta_{1} w
\end{array}\right), \quad\left(\begin{array}{c}
\hat{x}_{1}^{(2)} \\
\hat{x}_{2}^{(2)} \\
\hat{y}_{1}^{(2)} \\
\hat{y}_{2}^{(2)}
\end{array}\right)=\left(\begin{array}{c}
\eta_{1} w \\
\eta_{3} \eta_{1} w \\
-w \\
-\eta_{2} w
\end{array}\right) .
$$

In the new coordinates, the integral takes the form

$$
I_{p}=\int_{R_{3}} \int_{T_{1}^{h}} \eta_{1} w^{3} \sum_{i=1}^{2} H_{l o c}\left(\hat{x}^{(i)}, \hat{y}^{(i)}\right) k_{l o c}\left(\hat{x}^{(i)}, \hat{y}^{(i)}\right) d w d \eta
$$

In [12], [11], [15] it was shown that the integrand above is analytic and thus, can be approximated to any required accuracy with standard methods of properly chosen order.

It remains to consider the farfield case where $\overline{K_{1}} \cap \overline{K_{2}}=\emptyset$ holds. However, in this case the integrand is analytic and can be approximated by standard cubature methods with properly chosen order to any desired accuracy. In the following section we briefly sketch the cubature error analysis in appropriate norms.

\section{APPROXIMATION OF THE INTEGRALS AND CUBATURE ERROR ANALYSIS}

As mentioned above the integrands of the final integral representations are analytic and can be approximated by standard cubature formulae. The domains of integration are given by tensor products of the form $R_{d_{1}} \times T_{d_{2}}^{h}$ with $d_{1}+d_{2}=4$. We will use tensor product Gauß formulae for the integration over $R_{d_{1}}$ while for the variables contained in $T_{d_{2}}^{h}$ we employ abstract cubature formulae which are exact for polynomials of a predescribed degree. The reason for working with abstract formulae of a certain degree of exactness is that, especially for the $h$-version of the BEM, the use of Gauß-Legendre tensor formulae is sub-optimal on $T_{d_{2}}^{h}$ for practical problem sizes. However, the question which rule is optimal or convenient depends on the specific implementation and might be different for different situations. We refer to [2] and [17] for formulae which are exact for polynomials of certain orders on the domains $T_{d_{2}}^{h}$.

The estimates for the (local) cubature error are used to adapt the degrees of exactness to the consistency and stability requirements of the Galerkin discretization. Therefore, we will present the error estimates in terms of Sobolev norms since the consistency analysis for Galerkin methods is conveniently expressed in such norms.

In order to avoid too many technicalities, we restrict to triangulations which are quasiuniform and non-degenerate

$$
\begin{aligned}
& \max \{\operatorname{diam} K: K \in \tau\}:=h, \\
& \min \left\{\operatorname{diam} B_{K}: K \in \tau\right\} \geq \rho h,
\end{aligned}
$$

where $B_{K}$ denotes the largest ball contained in $K$ and the constant $\rho$ does not depend on $K$. 
Furthermore, we assume that the inverse estimate holds which we will use in the form that, for integers $l, m$ with $-\infty<l \leq m$, there exists a constant $C=C(l, m)$ such that, for all $v \in V_{k, p}(K)$, the estimate

$$
\|v\|_{W^{l, p}} \leq C h^{m-l+2 / p-1}\|v\|_{m}
$$

holds.

We will now come to the cubature error estimates. Let $D \subset \mathbb{R}^{d}$ be a domain. A cubature rule $M_{D}^{\gamma}$ has exactness degree $\gamma$ if, for all $f \in \prod_{\gamma}(D)$,

$$
E_{D}^{\gamma}[f]:=\left|\int_{D} f(x) d x-M_{D}^{\gamma}[f]\right|=0
$$

holds. The domains under consideration are of the form $R_{d_{1}} \times T_{d_{2}}^{h}$ with $d_{1}+d_{2}=4$. The notation $Q^{\gamma}[f]$ stands for the tensor cubature method $G_{R_{d_{1}}}^{\gamma_{1}} M_{T_{d_{2}}^{h}}^{\gamma_{2}}$ where $G_{R_{d_{1}}}^{\gamma_{1}}$ denotes the tensor Gauf formula composed of properly scaled one-dimensional Gauf-Legendre formulae $G_{(0,1)}^{\gamma_{1}}$ of order $\gamma_{1}$.

In [13] we have already considered the farfield case. Hence, we will concentrate here on the singular cases. We begin with the case of identical panels $K_{1}=K_{2}$. The representation (13) is approximated by $G_{R_{1}}^{\gamma_{1}} M_{T_{3}^{h}}^{\gamma_{2}}\left[g_{i}\right]$ where $g_{i}(\eta, w)$ is an abbreviation of the integrand of (13). The following theorem concerns the cubature error.

Theorem 4 The cubature error is given by $E_{R_{1} \times T_{3}^{h}}^{\gamma}:=\left|I_{i}-G_{R_{1}}^{\gamma_{1}} M_{T_{3}^{h}}^{\gamma_{2}}\left[g_{i}\right]\right|$. Let $0 \leq s, t$ and $p$ denote the polynomial degree of the trial space. In the case of $H(x, y)=A(x, y)$ we have

$$
E_{R_{1} \times T_{3}^{h}}^{\gamma} \leq C h\left((1+\lambda)^{-\gamma_{1}-1}\|u\|_{m_{1}}\|v\|_{m_{2}}+h^{\gamma_{2}+1-s-t}\|u\|_{p-s}\|v\|_{p-t}\right)
$$

with a constant $\lambda$ independent of the discretization parameters $h$ and $p$ and the orders $m_{j}$ of the operators $D_{j}$ defined at the beginning of the previous section.

For $H(x, y)=B(x, y)$ we obtain

$$
E_{R_{1} \times T_{3}^{h}}^{\gamma} \leq C h\left((1+\lambda)^{-\gamma_{1}-1}\|f\|_{m_{3}}\|v\|_{m_{4}}+h^{\gamma_{2}+1-t}\|f\|_{\gamma_{2}+1}\|v\|_{p-t}\right) .
$$

Proof. The proof is essentially the same as the proof of [15]. The only difference is the estimate of the trial functions $u$ and $v$ in suitable ellipses $\mathcal{E}$ of analyticity. The arguments of $u$ and $v$ range in intervals $I$ of length $h$. Hence, we obtain

$$
u(x)=\sum_{j=0}^{p} \sum_{|\alpha|=j} \frac{1}{\alpha !} u^{(\alpha)}(y)(x-y)^{\alpha}
$$

for any $y$ of the integration interval and $x \in \mathcal{E}$. In combination with the inverse estimate we obtain for $\operatorname{dist}(\partial \mathcal{E}, I)=O(1)$ :

$$
\max _{x \in \mathcal{E}}|u(x)| \leq \sum_{j=0}^{p} \frac{1}{j !}|u|_{W^{j, \infty}}(\operatorname{dist}(\partial \mathcal{E}, I))^{j} \leq C h^{-1}\|u\|_{p}
$$

and for $\operatorname{dist}(\partial \mathcal{E}, I)=O(h)$ :

$$
\max _{x \in \mathcal{E}}|u(x)| \leq C h^{-1} \sum_{j=0}^{p}|u|_{j} h^{j} \leq C h^{-1} \sum_{j=0}^{p} h^{-j}\|u\|_{0} h^{j}=C h^{-1}\|u\|_{0} .
$$


If $D_{j}$ is not the identity but a differentiating operator the estimate can be obtained in the same fashion but the inverse inequality has to be applied in the form of $|u|_{j} \leq$ $C h^{j-2}\|u\|_{1}$.

Next we consider the case that the panels $K_{1}$ and $K_{2}$ share exactly one edge. We approximate the integral in (14) by $G_{R_{2}}^{\gamma_{1}} M_{T_{2}^{h}}^{\gamma_{2}}\left[g_{e}\right]$ where $g_{e}(\eta, w)$ denotes the integrand of (14). The following theorem concerns the cubature error.

Theorem 5 Let $s, t \geq 0$. For $H=A$, the cubature error $E_{R_{2} \times T_{2}^{h}}^{\gamma}:=\left|I_{e}-G_{R_{2}}^{\gamma_{1}} M_{T_{2}^{h}}^{\gamma_{2}}\left[g_{e}\right]\right|$ can be estimated by

$$
E_{R_{2} \times T_{2}^{h}}^{\gamma} \leq C\left((1+\lambda)^{-\gamma_{1}-1}\|u\|_{m_{1}}\|v\|_{m_{2}}+h^{\gamma_{2}+1-s-t}\|u\|_{p-s}\|v\|_{p-t}\right)
$$

while for $H=B$ we obtain

$$
E_{R_{2} \times T_{2}^{h}}^{\gamma} \leq C\left((1+\lambda)^{-\gamma_{1}-1}\|f\|_{m_{3}}\|v\|_{m_{4}}+h^{\gamma_{2}+1-t}\|f\|_{\gamma_{2}+1}\|v\|_{p-t}\right) .
$$

Proof. The proof is essentially the same as worked out in [15] with the same modifications as pointed out in the previous proof.

It remains to discuss the case that $K_{1}$ and $K_{2}$ share exactly one vertex. We approximate the integral representation (15) by $G_{R_{3}}^{\gamma_{1}} M_{T_{1}^{h}}^{\gamma_{2}}\left[g_{p}\right]$ where $g_{p}$ denotes the integrand of (15). The cubature error is estimated in the following

Theorem 6 Let $s, t \geq 0$. For $H=A$, the cubature error $E_{R_{3} \times T_{1}^{h}}^{\gamma}:=\left|I_{p}-G_{R_{3}}^{\gamma_{1}} M_{T_{1}^{h}}^{\gamma_{2}}\left[g_{p}\right]\right|$ can be estimated by

$$
E_{R_{3} \times T_{1}^{h}}^{\gamma} \leq C\left((1+\lambda)^{-\gamma_{1}-1}\|u\|_{m_{1}}\|v\|_{m_{2}}+h^{\gamma_{2}+1-s-t}\|u\|_{p-s}\|v\|_{p-t}\right)
$$

while, for $H=B$, we get

$$
E_{R_{3} \times T_{1}^{h}}^{\gamma} \leq C\left((1+\lambda)^{-\gamma_{1}-1}\|f\|_{m_{3}}\|v\|_{m_{4}}+h^{\gamma_{2}+1-t}\|f\|_{\gamma_{2}+1}\|v\|_{p-t}\right)
$$

Proof. Again, the proof of this theorem is analogous as worked out in [15].

The regular integrals $a_{K}$ and $F_{K}$ are transformed to the scaled master element $T_{2}^{h}$ and approximated by a formula $Q_{T_{2}^{h}}^{\gamma}$ with exactness degree $\gamma$. The following theorem concerns the arising cubature error.

Theorem 7 Let $s, t \geq 0$. For the approximation of $a_{K}(u, v)$, the error $E_{T_{2}^{h}}^{\gamma}:=\left|a_{K}-Q_{T_{2}^{h}}\right|$ can be estimated by

$$
E_{T_{2}^{h}}^{\gamma} \leq C h^{1+\gamma-s-t}\|u\|_{p-s}\|v\|_{p-t}
$$

while for the approximation of $F_{K}(v)$ we get

$$
E_{T_{2}^{h}}^{\gamma} \leq C h^{1+\gamma-t}\|u\|_{p-t}\|f\|_{\gamma+1} .
$$

Proof. The proof of the first assertion is straightforward, while the second one was proved in [13, Theorem 6$]$.

Remark 8 The regularity assumptions on $f$ in the estimates above can be relaxed such that the required order of differentiability is independent of the degree of exactness (see [13, Theorem 5]). 


\section{ANALYSIS OF THE FULLY DISCRETE GALERKIN BEM}

The effect of numerical cubature is treated as an approximation of the discrete (bi-) linear forms. The cubature orders have to be chosen such that the asymptotic convergence of the Galerkin method is preserved. In [13], the effect of the local cubature error to the global discretization process was investigated thoroughly in view of stability and consistency. We can use these results directly to determine the required cubature orders. They will depend on the polynomial degree of approximation $p$, the step size $h$, the norm in which the error is measured, and are possibly different for different variables.

For simplicity, we assume here full regularity of the variational problem, i.e., the righthand side of (1) is such that

$$
\left\|u-u_{G}\right\|_{s_{1}-t} \leq C h^{p+1+t-s_{1}}\|f\|_{p+1-2\left(s_{1}+s_{2}\right)}
$$

is satisfied for $t \in\left[0, p+1-s_{1}\right]$. In the following, the orders $\gamma$ of the cubature formulae defined above are given such that the error $u-\tilde{u}_{G}$ converges with the same asymptotic rate as the Galerkin solution $u_{G}$. The notation $\lceil x\rceil$ stands for the smallest integer larger than or equal to $x$.

- The bilinear form $a_{K}$ is approximated by $Q_{K}^{\gamma}$ with

$$
\gamma=\left\lceil 2\left(p-s_{1}\right)\right\rceil
$$

- "Case of identical panels": the bilinear form $a_{K \times K}$ is approximated by $Q_{R_{1} \times T_{3}^{h}}^{\gamma}$ with

$$
\gamma_{1}=\left\lceil\left(p-2 s_{1}+t+m_{2}\right)|\log h|-1\right\rceil, \quad \gamma_{2}=\left\lceil 2\left(p-s_{1}\right)\right\rceil
$$

- "Case of a common edge": the bilinear form $a_{K_{x} \times K_{y}}$ is approximated by $Q_{R_{2} \times T_{2}^{h}}^{\gamma}$ with

$$
\gamma_{1}=\left\lceil\left(p+1-2 s_{1}+t+m_{2}\right)|\log h|-1\right\rceil, \quad \gamma_{2}=\left\lceil 2\left(p-s_{1}\right)\right\rceil .
$$

- "Case of a common point": the bilinear form $a_{K_{x} \times K_{y}}$ is approximated by $Q_{R_{3} \times T_{1}^{h}}$ with

$$
\gamma_{1}=\left\lceil\left(p+1-2 s_{1}+t+m_{2}\right)|\log h|-1\right\rceil, \quad \gamma_{2}=\left\lceil 2\left(p-s_{1}\right)\right\rceil .
$$

The degrees of exactness for the approximation of the linear form $F_{K}, F_{K_{1} \times K_{2}}$ are the same as the analog orders of the bilinear form. The cubature orders for the farfield cases can be found in [13].

\section{REFERENCES}

[1] ADAMS, R.: Sobolev Spaces. Academic Press, N.Y., 1975. 
[2] COOLS, R. and RABINOWITZ, P.: Monomial Cubature Rules since "Stroud": A Compilation. J. Comput. Appl. Math. 48:309-326, 1993.

[3] GENG, P., ODEN, J., and DEMKOWICZ L.: Solution of Exterior Acoustic Problems by the Boundary Element Method at High Wave Numbers, Error Estimation and Parallel Computation. Technical Report 95-09, TICAM, The University of Texas at Austin, 1995.

[4] HACKBUSCH, W. and SAUTER, S.A.: On the Efficient Use of the Galerkin Method to Solve Fredholm Integral Equations. Applications of Mathematics 38(4-5):301-322, 1993.

[5] HAN, H.: A Boundary Element Method for Signorini Problems in Three Dimensions. Numer. Math., 60:63-76, 1991.

[6] HAN, H.: The Boundary Element Method for Solving Variational Inequalities. Contemp. Math. 163, 1994.

[7] HAN, H.: The Boundary Integro-Differential Equations of Three-Dimensional Neuman Problem in Linear Elasticity. Numer. Math. 68(2):269-281, 1994.

[8] LAGE, C.: Software Development for Boundary Element Mehtods: Analysis and Design of Efficient Techniques (in German). PhD thesis, Lehrstuhl Prakt. Math., Universität Kiel, 1995.

[9] LAGE, C. and SAUTER, S.: Analysis and Software Design Aspects of Advanced Algorithms in Boundary Element Methods. Wiley, planned for 1996.

[10] NEDELEC, J.C.: Integral Equations with Non Integrable Kernels. Integral Equations Oper. Theory 5:562-572, 1982.

[11] V. PETERSDORFF, T. and SCHWAB, C.: Fully Discrete Multiscale Galerkin BEM. Technical Report 95-08, Seminar for Applied Mathematics, ETH Zürich, 1995.

[12] SAUTER, S.A.: Über die effiziente Verwendung des Galerkinverfahrens zur Lösung Fredholmscher Integralgleichungen. PhD thesis, Lehrstuhl. f. Prakt. Math., Universität Kiel, 1992.

[13] SAUTER, S.A. and KRAPP, A.: On the Effect of Numerical Integration in the Galerkin Boundary Element Method.Technical Report 95-4, Lehrstuhl Praktische Mathematik, Universität Kiel, Germany, 1995, to appear in Numer. Math.

[14] SAUTER, S.A. and SCHWAB, C.: Realization of hp-Galerkin BEM in 3-d. In HACKBUSCH, W. and WITTUM, G., editors, BEM : Implementation and Analysis of Advanced Algorithms, Proceedings of the 12th GAMM-Seminar, Kiel. Verlag Vieweg, 1996.

[15] SAUTER, S.A. and SCHWAB, C.: Quadrature for hp-Galerkin BEM in 3-d. Technical report 96-02, Seminar for Appl. Math., ETH Zürich, 1996.

[16] SCHWAB, C. and WENDLAND, W.: Kernel Properties and Representations of Boundary Integral Operators. Math. Nachr. 156:187-218, 1992.

[17] STROUD A.H.: Approximate Calculations of Multiple Integrals. Prentice Hall, Englewood Cliffs, 1973. 